\title{
Adaptive Mitosis Detection in Large in vitro Stem Cell Populations using Timelapse Microscopy
}

\author{
Tim Becker ${ }^{1,2,3}$, Daniel H. Rapoport ${ }^{1}$, Amir Madany Mamlouk ${ }^{2,3}$ \\ ${ }^{1}$ Fraunhofer Research Institution for Marine Biotechnology, Lübeck, Germany \\ ${ }^{2}$ Institute for Neuro- and Bioinformatics, University of Lübeck, Germany \\ ${ }^{3}$ Graduate School for Computing in Medicine and Life Science, University of Lübeck, \\ Germany \\ tim.becker@emb.fraunhofer.de
}

\begin{abstract}
Reliable analysis of adult stem cell populations in in vitro experiments still poses a problem on the way to fully understand the regulating mechanism of these cultures. However, it is essential in the use of cultivated endogenous cells in stem cell therapies. One crucial feature during automated analysis is clearly the robust detection of mitotic events. In this work, we use the fully labeled stem cell benchmark data set CeTReS I in order to evaluate different approaches of mitosis detection: a purely time line based approach; a feature-based motility detector; and a detector based on the cell morphology changes, for which we also propose an adaptive version. We demonstrate that the approach based on morphological change outperforms the static detectors. However, the set of optimal features is changing over time, and thus it is not surprising that a feature set adapted to the systems confluency shows the best performance.
\end{abstract}

\section{Introduction}

In order to fully understand the behavior and regulating mechanisms of in vitro stem cell populations, time-lapse phase contrast microscopy plays a major role. This technique enables the observation and analysis of a cell population in a completely label-free and noninvasive manner and allows a simultaneous processing of thousands of cells in vitro. An automated cell detection and tracking system facilitates the analysis of these data and represents a big improvement to the actual gold standard method: the tedious work of manual cell tracking. The key event in analyzing this data is cell division or mitosis. The mitosis is the most difficult part to detect as it implies a strong non-linearity or, in other words, new cells and tracking candidates are added to the scenario. However, an accurate knowledge of these events enables the reconstruction of cell lineages [1] as well as the complete characterization of cell populations including life time distributions, change of cell cycle length and (a)symmetry of cell divisions. Up to now, only a few approaches explicitly address the detection of mitotic events 
in unlabeled cell populations. Those that do typically use a spatio-temporal pattern that arises during mitosis. As an example, the radius of a cell can be used to determine such an event [2]. Other approaches use a characteristic change of different cell parameters, such as size, brightness and circularity [3].

\section{Material and Methods}

\subsection{Data}

All detection and tracking tasks were performed using the freely available data set CeTReS I [4]. This reference set contains two image series of adult stem cells from rat pancreas [5]. The first series consists of 209 images acquired every 15 minutes and shows the complete growth curve in detail: the initial lag, exponential and stationary phase (Fig. 1). This image series shows the biggest variety of cell characteristics and was therefore chosen for testing and evaluation.

\subsection{Cell Detection and Tracking}

Within this study, we use a straightforward algorithm to detect the cells automatically on the given microscope images. To get a consistent input, all images were preprocessed in two steps: first, an image normalization was used to expand the dynamic range of the data; second, the contrast was increased by performing a histogram equalization. To perform cell detection, each image is segmented using the Otsu intensity threshold. The result of this operation is a binary image that is further refined by morphological filters. This algorithm achieves a false acceptance rate (FAR) and false rejection rate (FRR) of $5 \%$ for cell detection and thus give a detection quality comparable with the state-of-the-art approaches [1]. The next task consists of following the cells over time, i.e. to

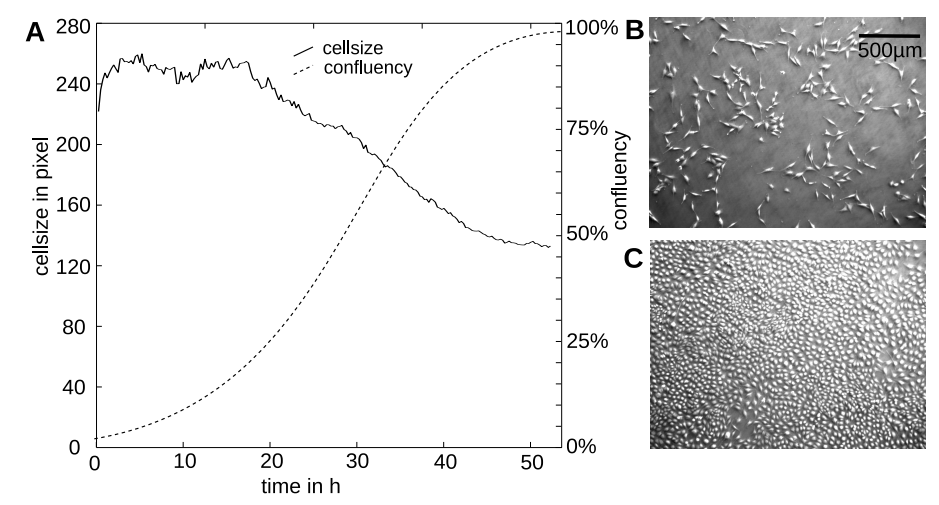

Fig. 1. Cell growths. (A) shows the population density and the change of cell size over time. The varying cell morphology is shown at $t=0 \mathrm{~h}(\mathrm{~B})$ and $t=52 \mathrm{~h}(\mathrm{C})$. 
track the cells. This task is performed using the overlap of the detected cell shapes in consecutive frames. As long as a unique cell successor can be determined, the cell shapes are used to construct cell paths $p$, i.e. a cell path consists of a series of a uniquely merged time series of cells. To correctly connect these paths and to reconstruct complete genealogical trees, it is essential that a path starts and ends with a mitosis.

\subsection{Mitosis Detection}

In general, there are two main approaches to detect mitoses: first, through temporal context; second, through a spatio-temporal pattern. Using the temporal context, a mitosis can be found as one cell (mother) divides into two cells (daughters). In a binary tree, this gives the typical Y-shape in a family tree. Furthermore, mitotic cells feature a characteristic spatio-temporal pattern (illustrated in Fig. 2). In particular, they contract, become smaller, rounder, appear brighter and decrease their velocity. Based on these criteria, four detectors were implemented for comparison:

- $Y$ detector: The naive approach classifies each path $p$ as mitotic if it can be linked to two successive cell paths and both have $p$ as their solely predecessor.

- Motility detector The second detector is motivated by Debeir et al. [2]. It simply classifies a cell path $p$ as being mitotic if both, size $S$ and circularity $C$ lie below a predefined threshold $\theta$.

- Morphology detector: The following set of cell features is computed for all detected single cells: perimeter $P$, cell area $A$, circularity $C$, average intensity (brightness) $B$ and the length $L$. The changes of these parameters, denoted as $d$, are calculated for all time points of a cell path $p$. A path $p$ is classified as being mitotic if the multidimensional normal distribution $\operatorname{Prob}(d)$ exceeds a threshold $\theta ; \operatorname{Prob}(d)$ is given by

$$
\operatorname{Prob}(d)=\frac{1}{\sqrt{(2 \pi)^{4} \operatorname{det}(\Sigma)}} \exp \left(\frac{-(d-\mu)^{T} \Sigma^{-1}(d-\mu)}{2}\right)
$$
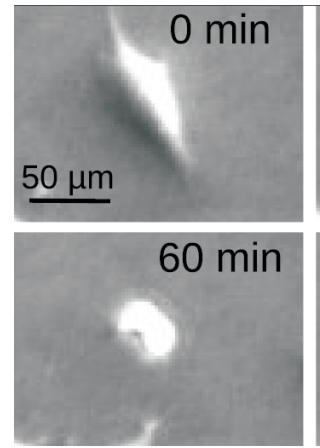
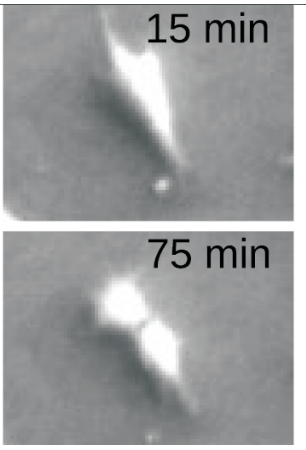
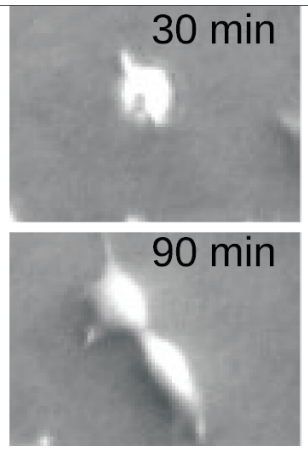
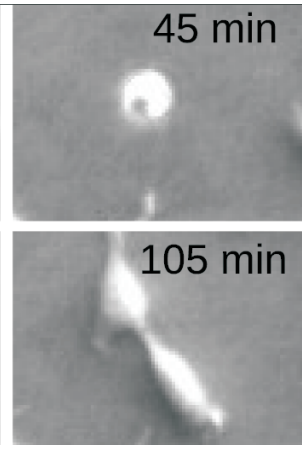

Fig. 2. Spatiotemporal pattern of mitosis. 
where $\Sigma$ denotes the covariance matrix and $\mu$ the mean feature difference vector. Values for $\mu$ and $\Sigma$ are trained using the reference data. These features combine to a set of potential classifiers $c(\omega, d)$, with $\omega \in\{0,1\}^{N}$ indicating which of the $N$ features are turned on or off.

- Adaptive morphology detector Finally, we propose a detector that substitutes $\omega$ in $c(\omega, d)$ by a time-dependent feature parameter $\omega(t)$. This appears to be a reasonable extension as single cell morphology is strongly changing over time due to population effects, e.g., confluency.

\section{Results}

The reference data CeTReS I was used to evaluate the proposed detectors. This set contains a total of 4689 cell paths and 2019 labeled mitotic events, of which $1903(95 \%)$ are successfully found by our proposed detection and tracking algorithm (2.2). To compare the different detection results, the false acceptance rate $(\mathrm{FAR}=$ specificity $)$ and false rejection rate $(\mathrm{FRR}=$ sensitivity $)$ for each detector was calculated with varying thresholds. In Figure 3(a), the ROC (Receiver Operator Characteristic) curves are shown for the different classifiers. The motility detection clearly performs worst. The motility detector is outperformed by the morphology detector using only size as a feature. Note that the motility variant use cell size as a static measure while the morphology detector is working on size differences. As shown, the combination of brightness and size parameter resulted in the best performance for the morphology detector. Figure 3(b) illustrates the behavior of the different detection strategies over time. The motility detection shows a strong decrease in performance leading to the worst result. Some of the morphology features show a contrary behavior: size has a decreasing performance while compactness has an increasing performace over time. This illustrates why the adaptive detection strategy performs best. From the biological point of view,

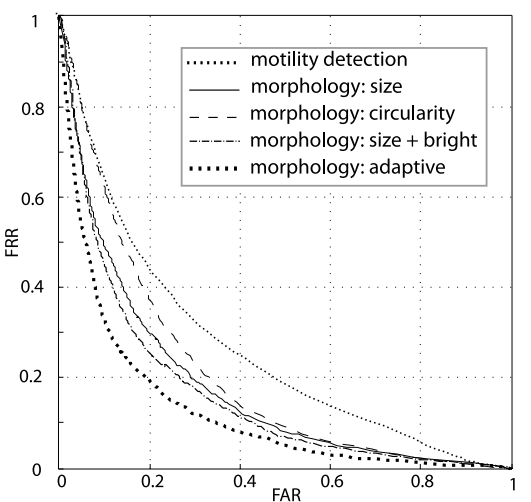

(a) ROC for different detections.

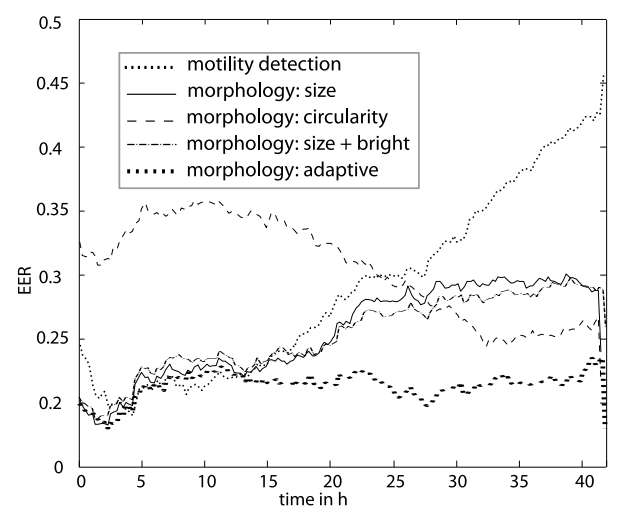

(b) EER over time

Fig. 3. Results for the different mitosis detections are shown as Receiver Operator Characteristic (ROC) and Equal Error Rate (EER) over time. 
Table 1. Number and and reliability (= true positves) of reconstructed complete paths (mitosis-mitosis) for different detections.

\begin{tabular}{llll}
\hline detection algorithm & Y detection & combined morphology & adaptive morphology \\
\hline complete paths & 1400 & 252 & 377 \\
reliability & $56.4 \%$ & $92.1 \%$ & $95.5 \%$ \\
\hline
\end{tabular}

the most important measure is the rate of correct detected complete paths, i.e. paths that describe complete cell cycles from the "birth" mitosis to the "end" mitosis. The results presented in table 1 show the number of detected paths and their reliability. To achieve the most reliable results, the $\mathrm{Y}$ detection was used to preselect possible mitosis candidates.

\section{Discussion}

In this paper, we present a mitosis detection based on the morphological change of the cells during division. The novelty of this algorithm lies in the fact that it adapts to the changes of cell appearance during culturing. Therefore, it outperforms other, non adaptive approaches like those of [2] or [3]. One has to bear in mind, that the different mitosis detection approaches have been tried on different time-lapse data. Therefore, a real quantitative comparison is not possible yet. We suggest to establish a common benchmark data set, like the CeTReS [4] timelapse series. Summarizing, the presented data illustrate the varying and inhomogeneous character of adult stem cell populations. This character has to be taken into account, when a fully automated cell tracking shall be used for future automated cell culture systems.

\section{References}

1. Li K, Chen M, Kanade T, et al. Cell population tracking and lineage construction with spatiotemporal context. Med Image Anal. 2008;12(1):546-66.

2. Debeir O, Milojevic D, Leloup T, et al. Mitotic tree construction by computer in vitro cell tracking: a tool for proliferation and motility features extraction. In: Proc EUROCON; 2005. p. 951-4.

3. yang F, michael a mackey, fiorenza ianzini, et al. Cell segmentation, tracking, and mitosis detection using temporal context. In: Proc MICCAI; 2005. p. 302-9.

4. Madany-Mamlouk A, Becker T, Rapoport D. A novel benchmark data set for adult stem cell tracking in time-lapse microscopy. In: Front Neur Conference Abstract: Neuroinformatics; 2009.

5. Kruse C, Kajahn J, Petschnik AE, et al. Adult pancreatic stem/progenitor cells spontaneously differentiate in vitro into multiple cell lineages and form teratomalike structures. Ann Anat. 2006;188:503-17. 